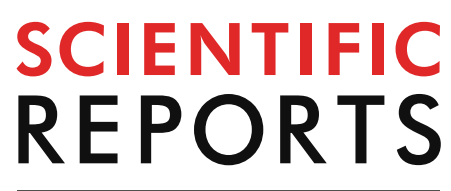

natureresearch

Check for updates

\title{
Gene polymorphisms and serum levels of sVEGFR-1 in patients with systemic lupus erythematosus
}

\author{
Zhi-Chao Yuan ${ }^{1}$, Wang-Dong Xu ${ }^{1 凶}$, Jia-Min Wang ${ }^{1}$, Qian Wu ${ }^{1}$, Jie Zhou ${ }^{1} \&$ An-Fang Huang ${ }^{2}$
}

Correlation between soluble vascular endothelial growth factor receptor-1 (sVEGFR-1) concentration, VEGFR1 gene polymorphisms and systemic lupus erythematosus (SLE) risk remains unclear. The present case-control study comprised 254 SLE patients, 385 other rheumatic diseases patients and 390 healthy controls. Serum levels of sVEGFR-1 were detected by enzyme-linked immunosorbent assay. Seven VEGFR1 genetic variants (rs2296188, rs9943922, rs2296283, rs7324510, rs9554322, rs9582036, rs9554320) were genotyped by KASP. Serum levels of sVEGFR-1 were up-regulated in SLE and positively correlated with disease activity. Furthermore, serum sVEGFR-1 presented a distinctive elevation in SLE in comparison with other rheumatic diseases. Frequencies of allele T of rs 2296283 and allele $G$ of rs9554322 were significant lower in SLE patients $(P=0.003, P=0.004)$. Frequencies of genotypes TT of rs 2296188 and rs 2296283 were declined in patients compared with healthy controls $(P=0.039, P=0.033)$. CC genotype of $\mathrm{rs} 7324510$ and $\mathrm{rs} 9582036$ was negatively correlated with SLE risk ( $O R=0.538, O R=0.508)$. Distribution of $G G, G C, G G+G C$ genotypes of rs9554322 were different between SLE patients and healthy controls $(P=0.027, P=0.036, P=0.010)$. Moreover, frequency of TC genotype of rs 7324510 was higher in SLE patients with lupus headache $\left(\chi^{2}=9.924, P=0.039\right)$ and frequency of TC genotype of rs9943922 was lower in patients with cylindruriain $\left(\chi^{2}=7.589, P=0.026\right)$. Frequencies of allele $C$ of rs7324510 and allele T of rs 9943922 were decreased in SLE patients with cylindruria and hypocomplementemia, respectively $\left(\chi^{2}=4.195, P=0.041, \chi^{2}=3.971, P=0.046\right)$. However, frequency of allele $C$ of rs 9554322 was increased in SLE patients with pyuria $\left(\chi^{2}=11.702\right.$, $P=0.001$ ). In addition, SLE patients carrying GG, GC, CC genotypes for rs9554322 had higher levels of serum sVEGFR-1. In conclusion, serum sVEGFR-1 was elevated in SLE patients and may be a disease marker. VEGFR1 gene polymorphisms related to risk of SLE in a Chinese Han population.

Systemic lupus erythematosus (SLE) is a chronic autoimmune disease with a heterogeneous organ involvement that hinges on multiple autoantibodies production. Considering the worldwide estimation of incidence of SLE, there was a regional variation of the disease. European countries had a lower incidence of SLE, whereas Asia, Australia and the Americas had higher incidence ${ }^{1}$. Gender difference also exists in SLE. It is known that occurrence of the disorder in women is more frequent than men, with a ratio of approximately $6: 1^{2}$. The aetiology of SLE has remained elusive, but genetic predisposition, environmental triggers and hormonal factors are demonstrated to involve in. A higher concordance rate in monozygotic twins than dizygotic twins and the high sibling recurrence risk ratio both support a strong heritability of $\mathrm{SLE}^{3}$. In the past decade, a variety of genomewide association studies (GWAS) have recognized over 40 SLE susceptibility loci existing in exons and introns ${ }^{4,5}$.

Soluble vascular endothelial growth factor receptor-1 (sVEGFR-1, also named as sFlt-1) is a nature vascular endothelial growth factor receptor (VEGFR) competitor. The human VEGFR1 gene is located in chromosome $13 q 12^{6,7}$. It was originally found to be expressed on vascular endothelial cells ${ }^{8}$, and then discovered expressed on smooth muscle cells ${ }^{9}$, monocytes ${ }^{10}$, trophoblasts $^{11}$, mesangial cells ${ }^{12}$ and osteoblasts ${ }^{13}$. sVEGFR-1 competes with signaling receptors of VEGF (VEGFR-1/VEGFR-2) by capturing their ligands ${ }^{14}$. It has a negative role in angiogenesis after binding to the VEGF. However, sVEGFR-1 interacts with endothelial cells components, showing an angiogenesis effect. Therefore, sVEGFR-1 exists multiple effects on vessel growth progression and may be a new

${ }^{1}$ Department of Evidence-Based Medicine, School of Public Health, Southwest Medical University, 1 Xianglin Road, Luzhou 646000, Sichuan, People's Republic of China. 'Department of Rheumatology and Immunology, Affiliated Hospital of Southwest Medical University, 25 Taiping Road, Luzhou 646000, Sichuan, People's Republic of China. ${ }^{\square}$ email: loutch123@163.com; loutch211@163.com 


\begin{tabular}{|c|c|c|c|c|c|c|c|}
\hline Characteristics & SLE & $\mathrm{HC}$ & RA & OA & gout & SS & AS \\
\hline $\begin{array}{l}\text { Female (\%)/ } \\
\text { male (\%) }\end{array}$ & $89.37 / 10.06$ & $91.79 / 8.21$ & $90.00 / 10.00$ & $86.96 / 13.04$ & $3.58 / 96.42$ & $85.19 / 14.81$ & $17.14 / 82.86$ \\
\hline Age (years) & $38(27.1-48.4)$ & $38(31.3-47.2)$ & $36(29.0-40.0)$ & $42(39.0-44.0)$ & $\begin{array}{l}38(26.50- \\
46.75)\end{array}$ & $44(44.0-46.7)$ & $37(32.0-45.5)$ \\
\hline $\begin{array}{l}\text { Lupus head- } \\
\text { ache, } \mathrm{n}(\%)\end{array}$ & $16(6.29)$ & - & - & - & - & - & - \\
\hline Vasculitis, n (\%) & $18(7.08)$ & - & - & - & - & - & - \\
\hline Arthritis, n (\%) & $106(41.73)$ & - & - & - & - & - & - \\
\hline Myositis, n (\%) & $12(4.72)$ & - & - & - & - & - & - \\
\hline Rash, n (\%) & $102(40.15)$ & - & - & - & - & - & - \\
\hline Alopecia, n (\%) & $64(25.19)$ & - & - & - & - & - & - \\
\hline $\begin{array}{l}\text { Oral ulcer, } \mathrm{n} \\
(\%)\end{array}$ & $28(11.02)$ & - & - & - & - & - & - \\
\hline Pleuritis, n (\%) & $23(9.06)$ & - & - & - & - & - & - \\
\hline $\begin{array}{l}\text { Pericarditis, } \\
\mathrm{n}(\%)\end{array}$ & $22(8.66)$ & - & - & - & - & - & - \\
\hline Fever, n (\%) & 45 (17.71) & - & - & - & - & - & - \\
\hline $\begin{array}{l}\text { Hypocom- } \\
\text { plementemia, } \\
\mathrm{n}(\%)\end{array}$ & $131(51.57)$ & - & - & - & - & - & - \\
\hline ds-DNA, n (\%) & $60(23.62)$ & - & - & - & - & - & - \\
\hline $\begin{array}{l}\text { Thrombocyto- } \\
\text { penia, } n(\%)\end{array}$ & $34(13.39)$ & - & - & - & - & - & - \\
\hline $\begin{array}{l}\text { Reduced leuko- } \\
\text { cyte, n (\%) }\end{array}$ & $26(10.24)$ & - & - & - & - & - & - \\
\hline $\begin{array}{l}\text { Cylindruria, } \\
\mathrm{n}(\%)\end{array}$ & $12(4.72)$ & - & - & - & - & - & - \\
\hline $\begin{array}{l}\text { Hematuria, } \\
\mathrm{n}(\%)\end{array}$ & $85(33.46)$ & - & - & - & - & - & - \\
\hline $\begin{array}{l}\text { Proteinuria, } \\
\mathrm{n}(\%)\end{array}$ & $115(45.28)$ & - & - & - & - & - & - \\
\hline Pyuria, n (\%) & $22(8.66)$ & - & - & - & - & - & - \\
\hline
\end{tabular}

Table 1. Main demographic and clinical characteristics in patients with SLE and control groups. SLE systemic lupus erythematosus, $H C$ healthy controls, $R A$ rheumatoid arthritis, $O A$ osteoarthritis, SS Sjögren's syndrome, $A S$ ankylosing spondylitis.

therapeutic target for VEGF-mediated pathological signaling. In systemic sclerosis (SSc) patients, lower serum level of sVEGFR-1 was detected in comparison with healthy controls ${ }^{15}$. In rheumatoid arthritis (RA) patients, expression of sVEGFR-1 was increased, correlating with VEGF concentration ${ }^{16}$. Serum level of sVEGFR-1 in patients with osteoarthritis (OA) was higher compared with that in non-arthritic controls ${ }^{16}$. VEGFR1 genetic variant associated with RA disease activity ${ }^{17}$. Collectively, sVEGFR-1 plays important roles in inflammatory and autoimmune diseases. To date, relationship of sVEGFR-1 and lupus is limited. What is the expression profile of sVEGFR-1 in lupus, if VEGFR1 gene polymorphisms relate to SLE risk needs to be discussed.

\section{Results}

Demographical and clinical characterization of study subjects. The demographical characteristics of the patients with SLE and controls are shown in Table 1. For SLE patients, age was 38 (27.1-48.4) years. The age was 36 (29.0-40.0) years for RA patients, 42 (39.0-44.0) years for OA patients, 38 (26.50-46.75) years for gout patients, 44 (44.0-46.7) years for Sjögren's syndrome (SS) patients, 37 (32.0-45.5) years for ankylosing spondylitis (AS) patients (Table 1). The Age of SLE group was matched among all control groups. For gender information about SLE group and controls, there was no gender difference between SLE patients and other study group excepting gout $(P<0.001)$ and AS $(P<0.001)$. In addition, the hypocomplementemia, proteinuria, arthritis and rash were the four dominant clinical characteristics in SLE patients (the proportion of 51.57\%, 45.28\%, $41.73 \%$ and $40.15 \%$, respectively). Other clinical information was contained in Table 1.

Correlation between serum levels of sVEGFR-1 and SLE. Concentration of sVEGFR-1 in patients with 61 SLE was higher in comparison with that in 94 healthy subjects $(17.738$ (7.604-26.286) vs 12.115 (8.655$12.115) \mathrm{ng} / \mathrm{ml}, P=0.015$, Fig. $1 \mathrm{~A}$ ). Correlation between sVEGFR-1 levels and SLE disease activity index (SLEDAI) was calculated, showing a statistically significant correlation $\left(r_{s}=0.557, P<0.001\right.$, Fig. 1B). Higher levels of serum sVEGFR-1 were discovered in active SLE patients than in less-active patients (7.968 (5.325-20.421) vs 22.435 (13.491-36.642) ng/ml, $P<0.001$, Fig. 1C). When discussing the association of serum levels of sVEGFR-1 and SLE clinical and laboratory characteristics, differences were existed in the patients with arthritis, alopecia, ds-DNA, hematuria $(P=0.001, P=0.021, P=0.012, P=0.017$, respectively, Fig. $1 \mathrm{D}-\mathrm{G})$. The other information 

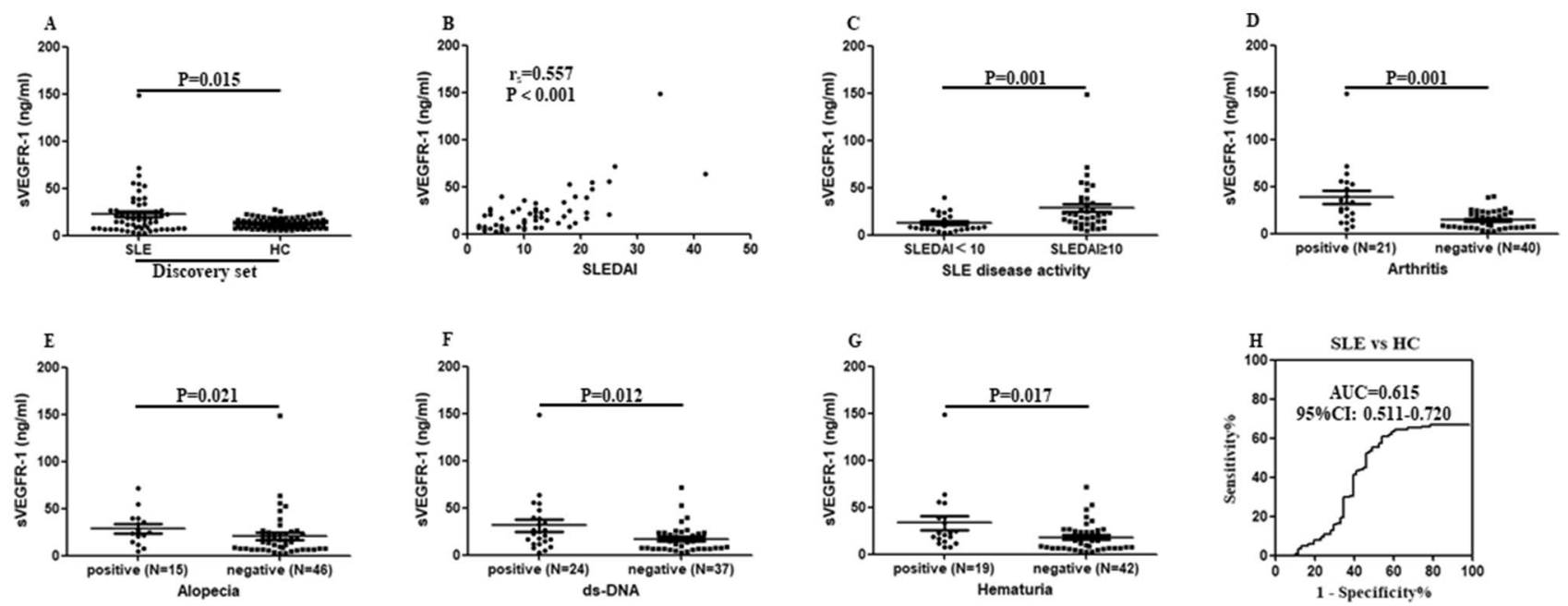

Figure 1. Serum levels of sVEGFR-1 in the discovery set. (A) Enzyme-linked immunosorbent assay was used to detect serum sVEGFR-1 levels in 61 systemic lupus erythematosus (SLE) patients and 94 healthy controls. Every symbol expresses an independent SLE patient and healthy control. (B) Correlation between SLEDAI and serum levels of sVEGFR-1. (C) Difference of serum levels of sVEGFR-1in SLE patients with less-active period and active period. (D-G) Difference of serum levels of sVEGFR-1 in several characteristics of SLE patients. (H) Potential of sVEGFR-1 as a disease marker for SLE, analyzed by receiver operating characteristic curve analysis.

\begin{tabular}{|l|l|l|l|l|}
\hline Clinical features & Positive n (\%) & Negative n (\%) & $\mathbf{Z}$ & $\boldsymbol{P}$ \\
\hline Vasculitis & $10(16.39)$ & $51(83.61)$ & 1.013 & 0.311 \\
\hline Arthritis & $21(34.43)$ & $40(65.57)$ & 3.931 & 0.001 \\
\hline Rash & $23(37.70)$ & $38(62.30)$ & 0.551 & 0.582 \\
\hline Alopecia & $15(24.59)$ & $46(75.41)$ & 2.311 & 0.021 \\
\hline Fever & $11(18.03)$ & $50(81.97)$ & 1.163 & 0.245 \\
\hline Hypocomplementemia & $21(34.43)$ & $40(65.57)$ & 1.199 & 0.230 \\
\hline ds-DNA & $23(37.70)$ & $38(62.30)$ & 2.188 & 0.029 \\
\hline Thrombocytopenia & $17(27.87)$ & $44(72.13)$ & 0.434 & 0.664 \\
\hline Hematuria & $19(31.15)$ & $42(68.85)$ & 2.383 & 0.017 \\
\hline Proteinuria & $31(50.82)$ & $30(49.18)$ & 1.774 & 0.076 \\
\hline SLEDAI & $38(62.30)$ & $23(37.70)$ & 3.199 & 0.001 \\
\hline
\end{tabular}

Table 2. Analysis of serum sVEGFR-1 levels in SLE by clinical features. $s$ VEGFR-1 soluble vascular endothelial growth factor receptor-1, SLE systemic lupus erythematosus, SLEDAI SLE disease activity index.

about the relationships between SVEGFR-1 and SLE clinical characteristics were exhibited in Table 2. Moreover, ROC curve showed that the AUC was 0.615 (95\% CI 0.511-0.720) (Fig. $1 \mathrm{H})$. These results suggested that serum sVEGFR-1 was up-regulated in SLE and positively correlated with disease activity. In validation set, 100 SLE patients and 385 disease controls (including RA, OA, gout, SS and AS) were compared to evaluate difference of serum levels of sVEGFR-1. Analysis indicated that serum concentration of sVEGFR-1 was significantly higher in SLE than that in RA, OA, gout, SS and AS groups (20.987 (13.080-40.885) vs 6.151 (3.614-12.134), 6.208 (4.129-10.293), 3.862 (2.790-5.817), 10.975 (5.513-15.963), 7.851 (5.268-7.851) ng/ml, all $P<0.001$, Fig. 2A-E). Serum sVEGFR-1 in SLE patients compared with that in RA, OA, gout, SS and AS patients showed AUC of $0.843,0.878,0.960,0.776,0.850$, respectively (Fig. 2F-J). Thus, these results revealed that serum sVEGFR-1 presented a distinctive elevation in SLE.

Polymorphisms of VEGFR1 and risk of SLE. To investigate whether polymorphisms of VEGFR1 gene affect SLE, seven SNPs were analyzed between SLE patients and healthy controls. No deviation from the HWE test was observed in patients and controls for each polymorphism $(P>0.05$, Table 3). Results found that SLE associated with genotypes or alleles of following SNPs: rs2296188, rs9943922, rs2296283, rs7324510, rs9554322, rs9582036. The genotypes and alleles frequencies were summarized in Table 4. Compared with healthy controls, frequency of rs2296283 allele T was strongly declined in SLE patients (OR=0.710, 95\% CI 0.567-0.891, $P=0.003)$. Similarly, frequency of rs 9554322 allele $\mathrm{G}$ was significantly associated with SLE $(\mathrm{OR}=0.667,95 \% \mathrm{CI}$ $0.468-0.901, P=0.004)$. As for frequencies of VEGFR1 genotypes, the most significant difference was observed in rs2296283, by which the genotype TT was dramatically decreased in SLE patients compared with healthy controls $(\mathrm{OR}=0.513,95 \% \mathrm{CI} 0.327-0.804, P=0.004)$. There were decreased frequencies of TT $+\mathrm{TC}$ in patients with 

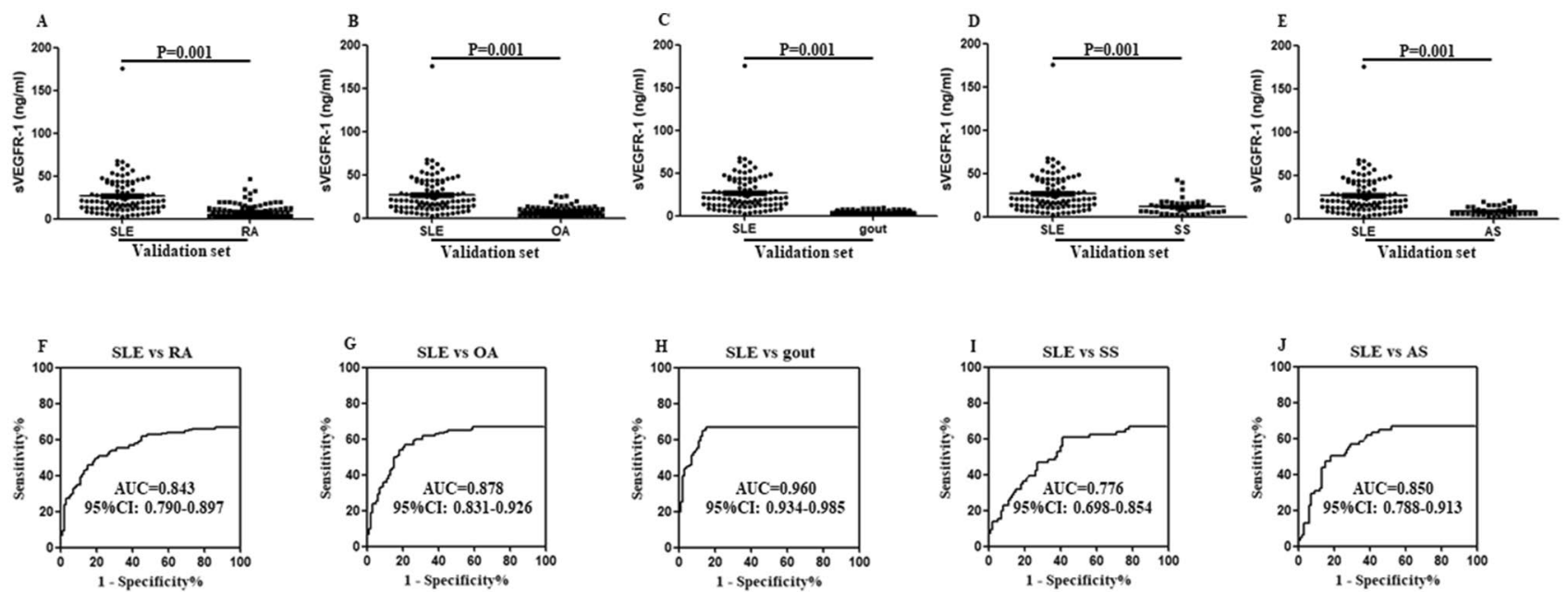

Figure 2. Serum levels of sVEGFR-1 in the validation set. (A-E) Differences of serum levels of sVEGFR-1 were tested between SLE and other rheumatic diseases (RA, OA, gout, SS and AS). (F-J) ROC curve analysis was performed to distinguish SLE from other rheumatic diseases.

\begin{tabular}{|l|l|l|l|l|}
\hline & SLE & & HC & \\
\hline rs2296188 & $\chi^{2}=0.001$ & $P=0.999$ & $\chi^{2}=3.434$ & $P=0.180$ \\
\hline rs9943922 & $\chi^{2}=0.032$ & $P=0.984$ & $\chi^{2}=1.199$ & $P=0.549$ \\
\hline rs2296283 & $\chi^{2}=1.053$ & $P=0.591$ & $\chi^{2}=0.368$ & $P=0.832$ \\
\hline rs7324510 & $\chi^{2}=0.037$ & $P=0.982$ & $\chi^{2}=4.662$ & $P=0.097$ \\
\hline rs9554322 & $\chi^{2}=0.009$ & $P=0.995$ & $\chi^{2}=0.167$ & $P=0.920$ \\
\hline rs9582036 & $\chi^{2}=0.276$ & $P=0.871$ & $\chi^{2}=5.685$ & $P=0.058$ \\
\hline rs9554320 & $\chi^{2}=0.453$ & $P=0.797$ & $\chi^{2}=0.136$ & $P=0.934$ \\
\hline
\end{tabular}

Table 3. The Hardy-Weinberg's expectation test in patients and controls of seven SNPs. SLE systemic lupus erythematosus, $H C$ healthy controls, SNPs single nucleotide polymorphisms.

SLE as compared with healthy controls $(\mathrm{OR}=0.588,95 \% \mathrm{CI} 0.394-0.876, P=0.009)$. Frequencies of all rs 9554322 genotypes (GG, GC, GG + GC) were lower in SLE group (OR=0.417, 95\% CI 0.192-0.907, $P=0.027$; OR = 0.694, 95\% CI 0.493-0.977, $P=0.036$; $O R=0.649,95 \%$ CI 0.468-0.901, $P=0.010$ ). For rs 2296188 and rs 9943922 , frequencies of TT were both lower in SLE patients when compared with controls (OR =0.578, 95\% CI 0.344-0.972, $P=0.039 ; \mathrm{OR}=0.619,95 \% \mathrm{CI} 0.398-0.962, P=0.033$ ). Moreover, we found marginal differences of genotype CC in rs7324510 and rs9582036 in SLE patients compared with healthy controls $(\mathrm{OR}=0.538,95 \%$ CI 0.296-0.976, $P=0.041 ; \mathrm{OR}=0.508,95 \% \mathrm{CI} 0.262-0.907, P=0.046$ ). Frequencies of rs 9554320 genotypes, although not statistically significant, were lower in SLE patients as compared with healthy subjects (data not show).

VEGFR1 haplotypes and SLE risk. In the present study, we exerted the haplotype analysis through constructing a block which comprised rs2296283, rs9943922 and $r$ 7324510 $\left(\mathrm{D}^{\prime}=0.915, \mathrm{r}^{2}=0.521 ; \mathrm{D}^{\prime}=0.716\right.$, $r^{2}=0.221 ; D^{\prime}=0.700, r^{2}=0.131$ ) (Fig. 3). Results revealed that the frequencies of CTA haplotype were higher in SLE patients in comparison with healthy controls $(\mathrm{OR}=1.435,95 \% \mathrm{CI} 1.137-1.810, P=0.002)$. Proportions of CTC and TTA haplotypes were fewer in SLE patients $(\mathrm{OR}=0.234,95 \%$ CI $0.081-0.674, P=0.003$; OR $=0.303$, 95\% CI 0.197-0.465, $P=0.001$ ). The other haplotypes were not figured out statistical significance (Table 5).

Association of VEGFR1 gene polymorphisms with clinical and laboratory features in

SLE. Given that SLE patients had diverse phenotypes and genetic predisposition, we explored association between VEGFR1 polymorphisms and clinical, laboratory features. The genotype and allele frequencies of VEGFR1 polymorphisms in SLE patients with different clinical manifestations were listed in Table 6. As the results shown, there was an increased frequency of TC genotype in patients with lupus headache in contrast to patients without this feature for $r 57324510\left(\chi^{2}=9.924, P=0.039\right)$. For rs9943922, TC genotype was different in patients with cylindruria as compared with patients without this feature $\left(\chi^{2}=7.589, P=0.026\right)$. Distribution of CC, CA, AA genotypes of rs9582036 and rs9554320 was different between SLE patients with and without pyuria $\left(\chi^{2}=14.437, P=0.003 ; \chi^{2}=15.074, P=0.001\right)$. In addition, $C$ allele frequency of rs7324510 was lower in SLE patients with hypocomplementemia comparing to those without $\left(\chi^{2}=4.195, P=0.041\right)$. T allele frequency of rs9943922 polymorphism was different between SLE patients with cylindruria and those without $\left(\chi^{2}=3.971\right.$, $P=0.046)$. It was found that the $C$ allele frequency of rs 9554322 was increased in SLE patients with pyuria 


\begin{tabular}{|c|c|c|c|c|c|}
\hline SNP & Genotype & SLE (N=254) n (\%) & Controls $(\mathrm{N}=390)$ n $(\%)$ & OR $(95 \% \mathrm{CI})$ & $P$ value \\
\hline \multirow{6}{*}{ rs 2296188} & TT & $25(9.8)$ & $62(15.9)$ & $0.578(0.344-0.972)$ & 0.039 \\
\hline & TC & $109(43.0)$ & $156(40.0)$ & $1.001(0.714-1.404)$ & 0.993 \\
\hline & $\mathrm{TT}+\mathrm{TC}$ & $134(52.8)$ & $218(55.9)$ & $0.881(0.641-1.211)$ & 0.434 \\
\hline & CC & $120(47.2)$ & $172(44.1)$ & Reference & \\
\hline & $\mathrm{T}$ & $159(31.3)$ & $280(35.9)$ & $0.814(0.614-1.032)$ & 0.089 \\
\hline & $\mathrm{C}$ & $349(68.7)$ & $500(64.1)$ & Reference & \\
\hline \multirow{6}{*}{ rs9943922 } & TT & $62(24.4)$ & $127(32.6)$ & $0.619(0.398-0.962)$ & 0.033 \\
\hline & TC & $125(49.2)$ & $178(45.6)$ & $0.891(0.601-1.321)$ & 0.565 \\
\hline & $\mathrm{TT}+\mathrm{TC}$ & $187(73.6)$ & $305(78.2)$ & $0.778(0.538-1.124)$ & 0.181 \\
\hline & $\mathrm{CC}$ & $67(26.4)$ & $85(21.8)$ & Reference & \\
\hline & $\mathrm{T}$ & $249(49.0)$ & $432(55.4)$ & $0.775(0.619-0.969)$ & 0.025 \\
\hline & $\mathrm{C}$ & $259(51.0)$ & $348(44.6)$ & Reference & \\
\hline \multirow{6}{*}{ rs2296283 } & TT & $79(31.1)$ & $154(39.5)$ & $0.513(0.327-0.804)$ & 0.004 \\
\hline & TC & $115(45.3)$ & $176(45.1)$ & $0.654(0.427-1.003)$ & 0.051 \\
\hline & $\mathrm{TT}+\mathrm{TC}$ & $194(76.4)$ & $330(84.6)$ & $0.588(0.394-0.876)$ & 0.009 \\
\hline & $\mathrm{CC}$ & $60(23.6)$ & $60(15.4)$ & reference & \\
\hline & $\mathrm{T}$ & $273(53.8)$ & $484(62.1)$ & $0.710(0.567-0.891)$ & 0.003 \\
\hline & $\mathrm{C}$ & $235(46.2)$ & $296(37.9)$ & Reference & \\
\hline \multirow{6}{*}{ rs7324510 } & $\mathrm{CC}$ & $17(6.7)$ & $46(11.8)$ & $0.538(0.296-0.976)$ & 0.041 \\
\hline & CA & $94(37.0)$ & $136(34.9)$ & $1.005(0.717-1.410)$ & 0.975 \\
\hline & $\mathrm{CC}+\mathrm{CA}$ & $111(43.7)$ & $182(46.7)$ & $0.887(0.646-1.220)$ & 0.460 \\
\hline & AA & $143(56.3)$ & $208(53.3)$ & Reference & \\
\hline & C & $128(25.2)$ & $229(29.3)$ & $0.810(0.629-1.044)$ & 0.103 \\
\hline & A & $380(74.8)$ & $551(70.7)$ & Reference & \\
\hline \multirow{6}{*}{ rs9554322 } & GG & $9(3.5)$ & $28(7.2)$ & $0.417(0.192-0.907)$ & 0.027 \\
\hline & GC & $77(30.3)$ & $144(36.9)$ & $0.694(0.493-0.977)$ & 0.036 \\
\hline & GG + GC & $86(33.8)$ & $172(44.1)$ & $0.649(0.468-0.901)$ & 0.010 \\
\hline & $\mathrm{CC}$ & $168(66.2)$ & 218 (55.9) & Reference & \\
\hline & G & $95(18.7)$ & $200(25.7)$ & $0.667(0.507-0.878)$ & 0.004 \\
\hline & C & $413(81.3)$ & $580(74.3)$ & Reference & \\
\hline \multirow{6}{*}{ rs9582036 } & $\mathrm{CC}$ & $13(5.1)$ & $37(9.4)$ & $0.508(0.262-0.907)$ & 0.046 \\
\hline & $\mathrm{CA}$ & $80(31.5)$ & $120(30.8)$ & $0.965(0.682-1.364)$ & 0.840 \\
\hline & $\mathrm{CC}+\mathrm{CA}$ & $93(36.6)$ & $157(40.2)$ & $0.857(0.619-1.188)$ & 0.354 \\
\hline & AA & $161(63.4)$ & $233(59.8)$ & Reference & \\
\hline & C & $106(20.8)$ & $193(24.8)$ & $0.802(0.613-1.049)$ & 0.108 \\
\hline & A & $402(79.2)$ & $587(75.2)$ & Reference & \\
\hline \multirow{6}{*}{ rs9554320 } & CC & $141(55.5)$ & $234(60.0)$ & $1.019(0.498-2.088)$ & 0.957 \\
\hline & CA & $100(39.4)$ & $134(34.3)$ & $1.263(0.607-2.632)$ & 0.532 \\
\hline & $\mathrm{CC}+\mathrm{CA}$ & $241(94.9)$ & $368(94.3)$ & $1.109(0.548-2.242)$ & 0.775 \\
\hline & AA & $13(5.1)$ & $22(5.6)$ & Reference & \\
\hline & C & $382(75.2)$ & $602(77.2)$ & $0.931(0.397-1.869)$ & 0.841 \\
\hline & A & $126(24.8)$ & $178(22.8)$ & Reference & \\
\hline
\end{tabular}

Table 4. Allele and genotype frequencies of seven SNPs in the VEGFR1 gene in SLE patients and healthy controls. SNP single nucleotide polymorphism, $S L E$ systemic lupus erythematosus, OR odds ratio, $C I$ confidence interval.

$\left(\chi^{2}=11.702, P=0.001\right)$. No significant association was found between the other SNPs and clinical, laboratory manifestations of SLE (Supplementary Table 1).

Variation of VEGFR1 and concentration of serum SVEGFR-1. To discuss possible significance of VEGFR1 SNPs on serum sVEGFR-1 concentration, serum sVEGFR-1 levels were compared according to genotypes of individual SNP. Analysis indicated that serum levels of sVEGFR-1 were significantly different among SLE patients carrying GG, GC, CC genotypes for rs9554322 ( $P=0.027$, Fig. 4A). The other SNPs were not related to serum levels of sVEGFR-1 (Fig. 4B-G). 


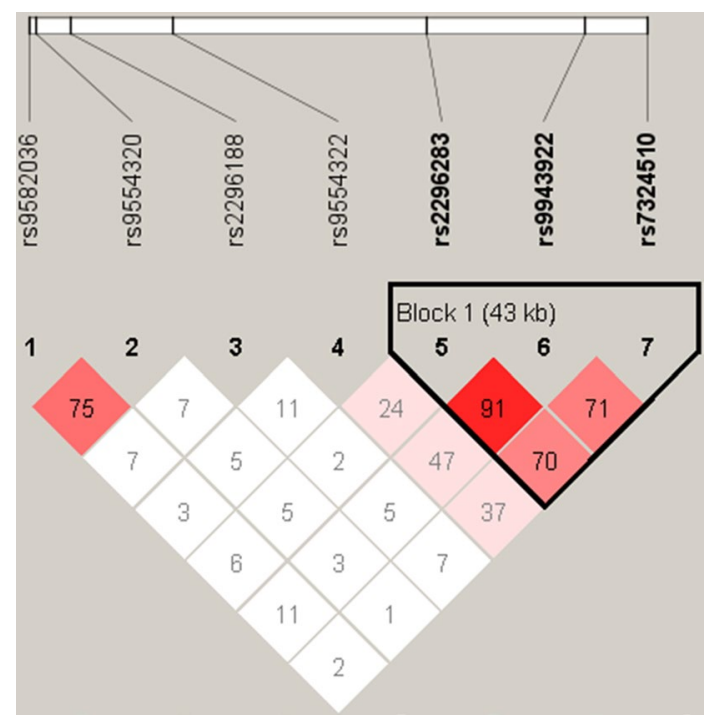

Figure 3. Linkage disequilibrium of seven SNPs. Seven SNPs (rs2296188, rs9943922, rs2296283, rs7324510, rs9554322, rs9582036, rs9554320) were selected in the study. The intensity of LD is reflected in the color and numeric value $\left(\mathrm{D}^{\prime}\right)$ of each box. Red and pink represent significant linkage, light blue and white indicate no linkage. D' value varies from 0 to 1 , and the 1 value represents the maximum linkage.

\begin{tabular}{|l|c|c|c|l|l|}
\hline Haplotype & SLE n (\%) & Controls n (\%) & $\chi^{\mathbf{2}}$ & P values & OR (95\% CI) \\
\hline CTA & $217.23(42.8)$ & $266.00(34.1)$ & 9.320 & 0.002 & $1.435(1.137-1.810)$ \\
\hline CTC & $4.03(0.8)$ & $25.54(3.3)$ & 8.557 & 0.003 & $0.234(0.081-0.674)$ \\
\hline TCA & $125.43(24.7)$ & $164.34(21.1)$ & 2.101 & 0.147 & $1.218(0.933-1.589)$ \\
\hline TCC & $119.83(23.6)$ & $173.21(22.2)$ & 0.252 & 0.615 & $1.071(0.820-1.398)$ \\
\hline TTA & $27.74(5.5)$ & $123.62(15.8)$ & 32.701 & 0.001 & $0.303(0.197-0.465)$ \\
\hline TTC & $0.00(0.0)$ & $20.84(2.7)$ & - & - & - \\
\hline CCA & $9.61(1.9)$ & $0.04(0.0)$ & - & - & - \\
\hline CCC & $4.13(0.8)$ & $6.41(0.8)$ & - & - & - \\
\hline
\end{tabular}

Table 5. Haplotype analysis between SLE patients and Healthy controls. Haplotype comprised rs2296283, rs9943922 and rs7324510. SLE systemic lupus erythematosus, OR odds ratio, CI confidence interval.

Statistical power. According to $\alpha=0.05, \mathrm{OR}=1.8$, and the MAF of each SNP, we calculated the statistical power. In the present study, the following powers were listed: 0.953 for rs2296188, 0.953 for rs $9943922,0.953$ for rs2296283, 0.948 for rs7324510, 0.951 for rs9554322, 0.941 for rs9582036, 0.948 for rs 9554320 , respectively.

\section{Discussion}

In the present study, we revealed that SVEGFR-1 serum levels associated with severity of SLE as well as VEGFR1 genetic variants existed in Chinese Han population. These findings added evidence that serum sVEGFR-1 and VEGFR1 gene were implicated in SLE pathogenesis. To our knowledge, this is the first study describing the network of serum sVEGFR-1, VEGFR1 gene and SLE risk. According to the findings, we observed that SLE patients showed higher serum levels of sVEGFR-1 which positively correlated with disease activity. Furthermore, genotype TT of rs2296188, TT of rs9943922, TT, TT + TC of rs2296283, CC of rs7324510, GG, GC, GG + GC of rs9554322 and CC of rs9582036 related to genetic susceptibility of SLE. SLE patients carrying rs2296283 G allele, rs9554322 T allele had decreased risk of SLE. Moreover, we found that VEGFR1 rs9554322 polymorphism may be a genetic factor for regulating sVEGFR-1 expression.

SLE is an autoimmune disease with a panel of clinical manifestations such as lupus nephritis that results from a variety of immunological and vascular abnormalities. Relationship between sVEGFR-1 and SLE is not clearly elucidated to date. VEGF is a crucial factor in circulating angiogenesis. It is able to modulate proliferation of endothelial cells, regulate chemotaxis, and capillary hyperpermeability in angiogenesis ${ }^{18}$. Studies indicated that sVEGFR-1 may be involved in SLE by affecting VEGF-mediated activation of angiogenesis. Several studies found that serum concentration of VEGF was elevated in SLE patients ${ }^{19-21}$. Renal glomerular microvasculature 


\begin{tabular}{|c|c|c|c|c|c|c|c|c|c|c|c|c|c|c|}
\hline \multirow{3}{*}{$\begin{array}{l}\text { Clinical } \\
\text { features }\end{array}$} & \multicolumn{7}{|l|}{ rs9943922 } & \multicolumn{7}{|l|}{ rs7324510 } \\
\hline & \multicolumn{3}{|c|}{ Genotype frequency, n (\%) } & \multirow[b]{2}{*}{$P$ value } & \multicolumn{2}{|c|}{$\begin{array}{l}\text { Allele frequency, } \\
\text { n (\%) }\end{array}$} & \multirow[b]{2}{*}{$P$ value } & \multicolumn{3}{|c|}{ Genotype frequency, $\mathbf{n}(\%)$} & \multirow[t]{2}{*}{$P$ value } & \multicolumn{2}{|c|}{$\begin{array}{l}\text { Allele frequency, } \\
\text { n (\%) }\end{array}$} & \multirow[b]{2}{*}{$P$ value } \\
\hline & TT & TC & $\mathrm{CC}$ & & $\mathrm{T}$ & C & & CC & CA & AA & & C & $\mathbf{A}$ & \\
\hline \multicolumn{15}{|c|}{ Lupus headache } \\
\hline Positive & $2(12.50)$ & $6(37.50)$ & $8(50.00)$ & 0.099 & $11(34.38)$ & $21(65.62)$ & 0.087 & $4(25.00)$ & $6(37.50)$ & $6(37.50)$ & 0.039 & $12(37.50)$ & $20(62.50)$ & 0.098 \\
\hline Negative & $60(25.21)$ & $\begin{array}{l}119 \\
(50.00) \\
\end{array}$ & $59(24.79)$ & & \begin{tabular}{|l}
238 \\
$(50.00)$ \\
\end{tabular} & $\begin{array}{l}238 \\
(50.00) \\
\end{array}$ & & $13(5.46)$ & $88(36.98)$ & $\begin{array}{l}137 \\
(57.56)\end{array}$ & & $\begin{array}{l}116 \\
(24.37) \\
\end{array}$ & $\begin{array}{l}360 \\
(75.63) \\
\end{array}$ & \\
\hline \multicolumn{15}{|c|}{ Hypocomplementemia } \\
\hline Positive & $31(23.66)$ & $65(49.62)$ & 35 (27.18) & 0.960 & \begin{tabular}{|l|}
128 \\
$(48.86)$
\end{tabular} & $\begin{array}{l}134 \\
(51.14)\end{array}$ & 0.940 & $5(3.82)$ & $46(38.12)$ & $80(61.06)$ & 0.095 & $56(21.37)$ & \begin{tabular}{|l|}
206 \\
$(78.63)$
\end{tabular} & 0.041 \\
\hline Negative & $31(25.20)$ & $60(48.78)$ & $32(26.02)$ & & $\begin{array}{l}121 \\
(49.19)\end{array}$ & $\begin{array}{l}125 \\
(50.81)\end{array}$ & & $12(9.76)$ & $48(39.02)$ & $63(51.22)$ & & $72(29.27)$ & \begin{tabular}{|l|}
174 \\
$(70.73)$
\end{tabular} & \\
\hline \multicolumn{15}{|c|}{ Cylindruria } \\
\hline Positive & $3(25.00)$ & $2(16.67)$ & $7(58.33)$ & 0.026 & $7(21.17)$ & $17(70.83)$ & 0.046 & $1(8.33)$ & $3(25.00)$ & $8(66.67)$ & 0.676 & $5(20.83)$ & $19(79.17)$ & 0.614 \\
\hline Negative & 59 (24.38) & $\begin{array}{l}123 \\
(50.83)\end{array}$ & $60(24.79)$ & & \begin{tabular}{|l|}
242 \\
$(50.00)$
\end{tabular} & $\begin{array}{l}242 \\
(50.00)\end{array}$ & & $16(6.61)$ & $91(37.60)$ & $\begin{array}{l}135 \\
(55.79)\end{array}$ & & $\begin{array}{l}123 \\
(25.41)\end{array}$ & \begin{tabular}{|l|}
361 \\
$(74.57)$
\end{tabular} & \\
\hline \multicolumn{15}{|l|}{ Pyuria } \\
\hline Positive & $2(9.09)$ & $13(59.09)$ & $7(31.82)$ & 0.216 & $17(0.386)$ & $27(61.36)$ & 0.150 & $1(4.54)$ & $7(31.82)$ & $14(63.64)$ & 0.744 & $9(20.45)$ & $35(79.55)$ & 0.448 \\
\hline Negative & $60(25.86)$ & $\begin{array}{l}112 \\
(48.28)\end{array}$ & $60(25.86)$ & & \begin{tabular}{|l|}
232 \\
$(50.00)$
\end{tabular} & $\begin{array}{l}232 \\
(50.00)\end{array}$ & & $16(6.90)$ & $87(37.50)$ & $\begin{array}{l}129 \\
(55.60)\end{array}$ & & $\begin{array}{l}119 \\
(25.65)\end{array}$ & \begin{tabular}{|l|}
345 \\
$(74.35)$ \\
\end{tabular} & \\
\hline \multirow{3}{*}{$\begin{array}{l}\text { Clinical } \\
\text { features }\end{array}$} & \multicolumn{7}{|l|}{ rs9582036 } & \multicolumn{7}{|l|}{ rs9554320 } \\
\hline & \multicolumn{3}{|c|}{ Genotype frequency, $\mathbf{n}(\%)$} & \multirow[b]{2}{*}{$P$ value } & \multicolumn{2}{|c|}{$\begin{array}{l}\text { Allele frequency, } \\
\text { n (\%) }\end{array}$} & \multirow[b]{2}{*}{$P$ value } & \multicolumn{3}{|c|}{ Genotype frequency, $\mathbf{n}(\%)$} & \multirow[b]{2}{*}{$P$ value } & \multicolumn{2}{|c|}{$\begin{array}{l}\text { Allele frequency, } \\
\text { n (\%) }\end{array}$} & \multirow[b]{2}{*}{$P$ value } \\
\hline & $\mathrm{CC}$ & CA & AA & & C & A & & CC & CA & AA & & C & $\mathbf{A}$ & \\
\hline \multicolumn{15}{|c|}{ Lupus headache } \\
\hline Positive & $1(6.25)$ & $4(25.00)$ & $11(68.75)$ & 0.840 & $6(18.75)$ & $26(81.25)$ & 0.761 & $8(50.00)$ & $7(43.75)$ & $1(6.25)$ & 0.896 & $23(71.88)$ & $9(28.13)$ & 0.653 \\
\hline Negative & $12(5.04)$ & $76(31.93)$ & $\begin{array}{l}150 \\
(63.03)\end{array}$ & & $\begin{array}{l}100 \\
(21.01)\end{array}$ & $\begin{array}{l}376 \\
(78.99)\end{array}$ & & $\begin{array}{l}133 \\
(55.88)\end{array}$ & $93(39.08)$ & $12(5.04)$ & & $\begin{array}{l}359 \\
(75.42)\end{array}$ & $\begin{array}{l}117 \\
(24.58)\end{array}$ & \\
\hline Hypocom & lementemia & & & & & & & & & & & & & \\
\hline Positive & $4(3.05)$ & $44(33.59)$ & $83(63.36)$ & 0.269 & $53(20.23)$ & $\begin{array}{l}209 \\
(79.77) \\
\end{array}$ & 0.715 & $71(54.20)$ & $54(41.22)$ & $6(4.58)$ & 0.790 & \begin{tabular}{|l|}
196 \\
$(74.81)$ \\
\end{tabular} & $66(25.19)$ & 0.835 \\
\hline Negative & $9(7.32)$ & $36(29.27)$ & $78(63.41)$ & & $53(21.54)$ & $\begin{array}{l}193 \\
(78.46)\end{array}$ & & $70(56.91)$ & $46(37.40)$ & $7(5.69)$ & & $\begin{array}{l}186 \\
(75.61)\end{array}$ & $60(24.39)$ & \\
\hline Cylindru & & & & & & & & & & & & & & \\
\hline Positive & $0(0.00)$ & $6(50.00)$ & $6(50.00)$ & 0.306 & $7(29.17)$ & $17(70.83)$ & 0.305 & $6(50.00)$ & $6(50.00)$ & $0(0.00)$ & 0.584 & $16(66.67)$ & $8(33.33)$ & 0.322 \\
\hline Negative & $13(5.37)$ & $74(30.58)$ & $\begin{array}{l}155 \\
(64.05)\end{array}$ & & 99 (20.45) & $\begin{array}{l}385 \\
(79.55)\end{array}$ & & $\begin{array}{l}135 \\
(55.79)\end{array}$ & $94(38.84)$ & $13(5.37)$ & & \begin{tabular}{|l}
366 \\
$(75.62)$
\end{tabular} & \begin{tabular}{|l|}
118 \\
$(24.38)$
\end{tabular} & \\
\hline Pyuria & & & & & & & & & & & & & & \\
\hline Positive & 4 (18.18) & $11(50.00)$ & $7(31.82)$ & 0.003 & $18(40.91)$ & $26(59.09)$ & 0.001 & $5(22.73)$ & $13(59.09)$ & $4(18.18)$ & 0.001 & $24(54.54)$ & $20(45.45)$ & 0.150 \\
\hline Negative & $9(3.88)$ & 69 (29.74) & \begin{tabular}{|l}
154 \\
$(66.38)$
\end{tabular} & & 88 (18.97) & $\begin{array}{l}376 \\
(81.03)\end{array}$ & & \begin{tabular}{|l}
136 \\
$(58.62)$ \\
\end{tabular} & $87(37.50)$ & $9(3.88)$ & & \begin{tabular}{|l}
358 \\
$(77.16)$ \\
\end{tabular} & $\begin{array}{l}106 \\
(22.84) \\
\end{array}$ & \\
\hline
\end{tabular}

Table 6. Analysis of VEGFR1 gene polymorphisms in SLE by clinical, laboratory features. SLE systemic lupus erythematosus.

is susceptible to local VEGF-A ${ }^{22}$. VEGF mediates the glomerular endothelial cell proliferation and survival in damaged glomerular capillaries ${ }^{23}$. The dysregulation of VEGF is involved in initiation of glomerular injury ${ }^{24}$. Therefore, regulation of VEGF is important in lupus nephritis development. sVEGFR-1 was encoded by a specifically spliced form of VEGFR-1 mRNA. It is comprised of $656 \mathrm{~N}$-terminal residues of the receptor, with a specific 30 amino acid tail at C-terminus. In SLE patients, plasma levels of sVEGFR-1 were found higher in patients with active lupus nephritis ${ }^{25}$. Similarly, in our study, we found that serum sVEGFR-1 was up-regulated in SLE and positively correlated with disease activity. In the inflammatory circumstance, sVEGFR-1 was secreted by activated monocytes ${ }^{25}$. High concentration of sVEGFR-1 antagonized the protective effect of VEGF, aggravating endothelial cell damage ${ }^{26}$. Therefore, it is hypothesized that sVEGFR-1 was up-regulated in the inflammatory circumstance of SLE, which bound to VEGF, leading to endothelial cell damage, further promoting inflammation in SLE.

Concerning VEGFR1 gene polymorphisms, our findings found that six SNPs (rs2296188, rs9943922, rs2296283, rs7324510, rs9554322, rs9582036) correlated with risk of SLE. It is worthy that rs9554322 and rs9582036 was the first time found to relation with SLE. Genotypes of GG, GC and GG + GC in rs9554322 were significantly lower in patients than in healthy controls, suggesting that rs 9554322 polymorphisms may negatively correlate with the risk of SLE in Chinese Han population $(\mathrm{OR}=0.417, \mathrm{OR}=0.694, \mathrm{OR}=0.649)$. Genotype $\mathrm{CC}$ of rs9582036 was related to SLE risk $(\mathrm{OR}=0.508)$. It is possible that regulatory, structural or quantitative polymorphisms at the VEGFR1 locus may affect VEGF signaling pathway and enhance susceptibility to some 

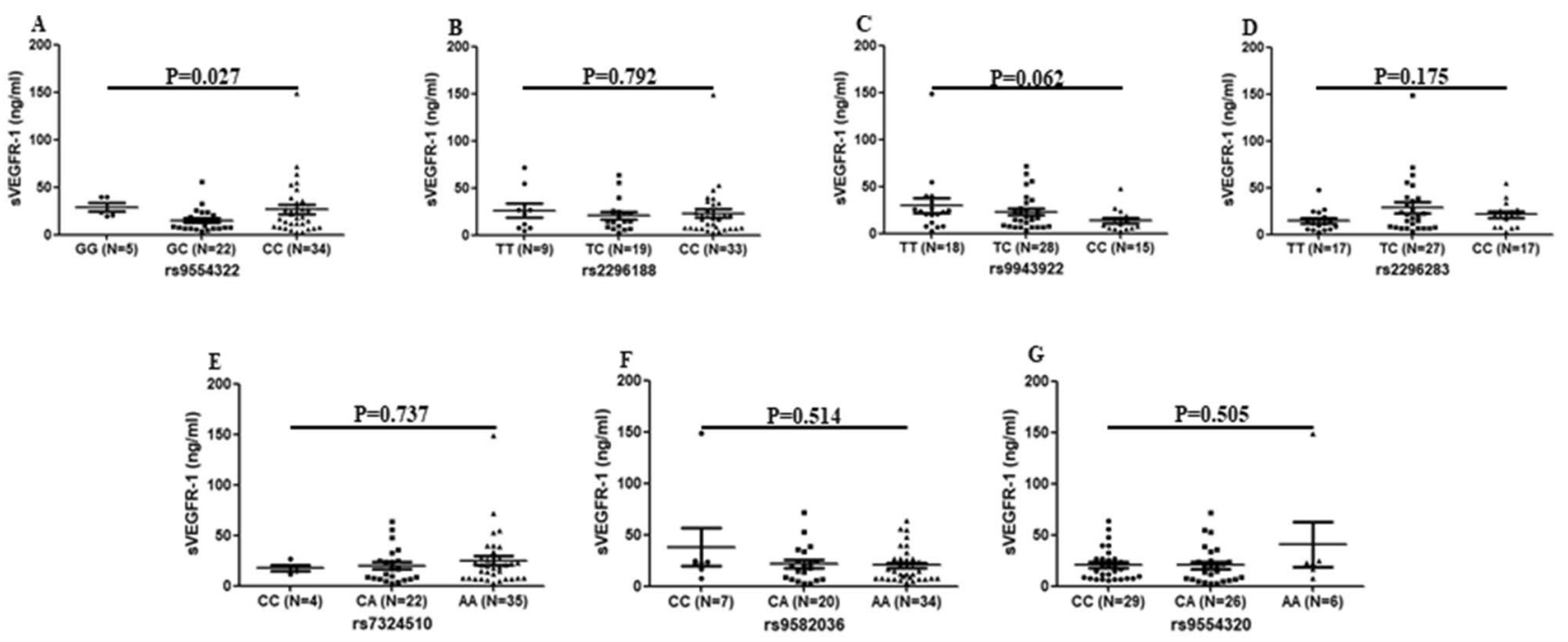

Figure 4. Genotypes of VEGFR1 gene and serum sVEGFR-1. Serum levels of sVEGFR-1 were examined by ELISA and $s$ VEGFR1 gene polymorphisms (rs2296188, rs9943922, rs2296283, rs7324510, rs9554322, rs9582036, rs9554320) were genotyped by PCR in SLE patients $(\mathrm{N}=61)$. (A) SLE patients carrying GG genotypes $(\mathrm{N}=5)$ of rs9554322 in VEGFR1 gene showed higher expression of sVEGFR-1. (B-G) Protein concentrations of sVEGFR-1 were not related to genotypes of rs2296188, rs9943922, rs2296283, rs7324510, rs9582036 and rs9554320 in VEGFR1 gene. Comparison of sVEGFR-1 values among three groups was performed by the Kruskal-Wallis test.

angiogenic conditions. In our study, rs2296283 located in the functional 3'-UTR region and other three SNPs (rs2296188, rs9943922 and rs7324510) were within the introns. In a Polish study, there was no statistic difference in allele or genotypes frequencies of rs 2296283 between RA patients and healthy controls ${ }^{17}$. On the contrary, we obtained a statistical difference in the frequencies of genotype TT and TT + TC for rs2296283 in SLE patients $(\mathrm{OR}=0.513, \mathrm{OR}=0.588)$. Compared with the Polish study, we further observed statistic differences in genotype TT of rs2296188, TT of rs9943922 and CC of rs7324510 ( $\mathrm{OR}=0.578, \mathrm{OR}=0.619, \mathrm{OR}=0.538)$, which were encoded in the introns. Therefore, rs 2296283 located in the functional region of VEGFR1 gene may influence the mRNA translation and stability through regulating polyadenylation, miRNA-mRNA and protein-mRNA interactions. However, further study needs to verify the effect of rs 2296283 within the functional region. The significance of SNPs located in non-coding region of VEGFR1 (introns) were not clear. Several studies showed that polymorphisms in non-coding region of VEGFR1 possibly silenced or enhanced the transcriptional activity of sVEGFR-1 ${ }^{27,28}$. Thus, it is postulated that polymorphisms in the non-coding region may implicate in selective splicing of RNA and promote the transcriptional activity of target protein. It is well known that SNP is the predominant pattern in the genomic DNA sequence variation. The majority of SNPs affected the gene expression instead of the protein composition. In agreement with previous studies, our results exhibited that six of seven SNPs (including rs2296188, rs9943922, rs2296283, rs7324510, rs9582036, rs9554320) were not related with sVEGFR-1 levels. Several GWAS studies indicated that although most of SNPs did not possess the direct ability to change the gene expression, these SNPs sometimes served for the functional counterparts which regulated gene expression and protein assemble ${ }^{29,30}$. Thus, we hypothesized that the six SNPs selected in this study may not directly influence sVEGFR-1 concentration in SLE patients. On the other side, serum levels of sVEGFR-1 were different among rs9554322 genotypes $(P=0.029)$. SLE patients with rs9554322 GG genotype had higher sVEGFR-1 protein levels, suggesting that VEGFR1 rs9554322 G/C genetic variant may contribute to abnormal sVEGFR-1 serum levels. However, what is the exact role of polymorphisms in VEGFR1 gene, sVEGFR-1 protein expression needs to be discussed and whether the selected SNPs in the current study can affect VEGFR1 gene, sVEGFR-1 protein expression needs to be conducted by functional study in the future.

There are several limitations in this study. First, the sample size in our study is relatively limited, where cases were recruited from two hospitals. Considering the large number of Chinese Han population, larger scale and multi-center studies are needed in the future. Second, the clear mechanism of sVEGFR-1 involves in SLE pathogenesis needs to discuss.

In summary, the present study showed that serum levels of sVEGFR-1 were elevated in SLE patients, may be a disease marker, and VEGFR1 gene polymorphisms related to risk of SLE in a Chinese Han population.

\section{Materials and methods}

Study subjects. Case-control studies were conducted for sVEGFR-1 serum levels and gene polymorphisms. A total of 254 SLE patients, 385 other rheumatic diseases patients and 390 healthy controls were recruited in the present study. Test for serum levels of sVEGFR-1was performed in two stages. The discovery set included 61 SLE and 94 healthy controls, and the validation set comprised another independent 100 SLE and 385 SLE-free disease controls [100 RA, 100 OA, 100 gout, 44 Sjögren's syndrome (SS) and 41 ankylosing spondylitis (AS)]. The study of gene polymorphisms consisted of 254 SLE and 390 healthy controls. All patients were recruited 
from the Department of Rheumatology and Immunology, Affiliated Hospital of Southwest Medical University and Affiliated Minda Hospital of Hubei Minzu University, classified by 1997 American College of Rheumatology (ACR) revised criteria for $\mathrm{SLE}^{31}, 1987$ ACR revised criteria for $\mathrm{RA}^{32}$, Osteoarthritis Criteria Subcommittee of the American Rheumatism Association (ARA) criteria for $\mathrm{OA}^{33}, 1977$ ARA criteria for gout ${ }^{34}$, American-European classification criteria for $\mathrm{SS}^{35}$ and Modified New York criteria for $\mathrm{AS}^{36}$. The SLE disease activity index (SLEDAI) was calculated to evaluate the disease activity of SLE patients ${ }^{37}$. Based on the SLEDAI score, the disease activity of SLE patients were divided into less-active period (SLEDIA $<10$ ) and active period (SLEDIA $\geq 10)$. Age and sex matched healthy controls were selected from Jiangyang district center for disease control and prevention in Luzhou, having no history of SLE and other inflammatory autoimmune diseases. All the participants were Chinese Han origin. The Medical Ethics Committee of Southwest Medical University approved our study protocol. Written informed consent was obtained from each subject. Blood samples were collected from patients and healthy controls.

SNP selection. A systemic search for previous literature about VEGFR1 gene was performed. Based on the 1,000-genome project (https://www.ncbi.nlm.nih.gov/variation/tools/1000genomes/), all candidate SNPs complied with following screening criteria: pairwise tagging of HapMap population with $\mathrm{r}^{2} \geq 0.8$; a minor allele frequency (MAF) $\geq 5 \%$; Chinese Han Beijing (CHB) ethnicity. At last, seven SNPs including rs2296188, rs9943922, rs2296283, rs7324510, rs9554322, rs9582036, rs9554320 were selected.

DNA extraction and genotyping analysis. Peripheral blood was collected from ulnar veins in the fasting and clearheaded state. Samples were centrifuged and serum was stored at $-80^{\circ} \mathrm{C}$ until analysis. Genomic DNA was extracted utilizing TIANamp Blood DNA kits (TIANGEN, Beijing, China) in line with manufacturer's instructions. VEGFR1 genotyping reactions were completed by Gene Company using KASP (Gene Company, Shanghai, China). Information of KASP primers (Primer_AlleleFAM, Primer_AlleleHEX and Primer_Common) was listed in Supplementary Table 2. To prove the reliability of genotyping results, five percent of the whole samples were repeatedly genotyped. Concordance rate of the repeated cases performed $100 \%$, demonstrating that the results were reliable in this study.

sVEGFR-1 protein measurement. sVEGFR-1 protein levels of SLE patients and control groups were assessed by enzyme-linked immunosorbent assay (ELISA) kits (Cusabio, Houston, USA) in accordance with the manufacturer's protocol. All samples were measured in duplicates and plates were read automatically at an absorbance of $450 \mathrm{~nm}$ using LT-4000MS reader (Labtech International Ltd, East Sussex, UK). Concentration was calculated on the basis of a linear standard curve. The detection limit was $0.039 \mathrm{ng} / \mathrm{ml}$.

Statistical analysis. Data was performed by Statistical Package for the Social Sciences (SPSS Inc., Chicago, version 17.0). Categorical data were expressed as frequency and percentage. According to Shapiro-Wilk test, measurement data were expressed by mean \pm standard deviation (SD) when it was normally distributed or median (inter-quartile range) when it was not normally distributed. For comparison of genotype and allele distribution between cases and controls, chi-squared or Fisher's exact test were used as appropriate. Odds ratio (OR) and 95\% confidence interval (CI) were analyzed by logistic regression model. Relationship between two variables was evaluated using Spearman's rank test. Area of receiver operating characteristic (ROC) curve evaluated the specificity and sensitivity of predictive power of sVEGFR-1 in SLE. Hardy-Weinberg equilibrium (HWE) of genotypes in patients and healthy controls was estimated by chi-squared test. HaploView 4.1 software was used to analyze linkage disequilibrium (LD). VEGFR1 haplotypes were assessed by using online software SHEsis (https://analysis.bio-X.cn). Statistical power was assessed (https://biostat.mc.vanderbilt.edu/wiki/Main/ PowerSampleSize). $P$ value lower than 0.05 was significant.

\section{Data availability}

All data supporting the results of this study are available in the article and supplementary information files or are available from the authors upon request.

Received: 10 January 2020; Accepted: 8 July 2020

Published online: 14 September 2020

\section{References}

1. Rees, F., Doherty, M., Grainge, M. J., Lanyon, P. \& Zhang, W. The worldwide incidence and prevalence of systemic lupus erythematosus: a systematic review of epidemiological studies. Rheumatology (Oxford) 56, 1945-1961 (2017).

2. Rees, F. et al. The incidence and prevalence of systemic lupus erythematosus in the UK, 1999-2012. Arthritis Rheum. 75, 136-141 (2016).

3. Alarcon-Segovia, D. et al. Familial aggregation of systemic lupus erythematosus, rheumatoid arthritis, and other autoimmune diseases in 1,177 lupus patients from the GLADEL cohort. Arthritis Rheum. 52,1138-1147 (2005).

4. Cui, Y., Sheng, Y. \& Zhang, X. Genetic susceptibility to SLE: recent progress from GWAS. J. Autoimmun. 41, 25-33 (2013).

5. Farh, K. K. et al. Genetic and epigenetic fine mapping of causal autoimmune disease variants. Nature 518, 337-343 (2015).

6. McGinnis, R. et al. Variants in the fetal genome near FLT1 are associated with risk of preeclampsia. Nat. Genet. 49, 1255-1260 (2017).

7. Wang, H. et al. Genetic variant in the 3'-untranslated region of VEGFR1 gene influences chronic obstructive pulmonary disease and lung cancer development in Chinese population. Mutagenesis 29, 311-317 (2014).

8. Barleon, B. et al. Differential expression of the two VEGF receptors flt and KDR in placenta and vascular endothelial cells. J. Cell. Biochem. 54, 56-66 (1994). 
9. Ishida, A. et al. Expression of vascular endothelial growth factor receptors in smooth muscle cells. J. Cell. Physiol. 188, 359-368 (2001).

10. Barleon, B. et al. Migration of human monocytes in response to vascular endothelial growth factor (VEGF) is mediated via the VEGF receptor flt-1. Blood 87, 3336-3343 (1996).

11. Ahmed, A., Dunk, C., Kniss, D. \& Wilkes, M. Role of VEGF receptor-1 (Flt-1) in mediating calcium-dependent nitric oxide release and limiting DNA synthesis in human trophoblast cells. Lab. Invest. 76, 779-791 (1997).

12. Amemiya, T. et al. Vascular endothelial growth factor activates MAP kinase and enhances collagen synthesis in human mesangial cells. Kidney Int. 56, 2055-2063 (1999).

13. Gerber, H. P. et al. VEGF couples hypertrophic cartilage remodeling, ossification and angiogenesis during endochondral bone formation. Nat. Med. 5, 623-628 (1999).

14. Kendall, R. L. \& Thomas, K. A. Inhibition of vascular endothelial cell growth factor activity by an endogenously encoded soluble receptor. Proc. Natl. Acad. Sci. U. S. A. 90, 10705-10709 (1993).

15. Avouac, J. et al. Angiogenesis in systemic sclerosis: impaired expression of vascular endothelial growth factor receptor 1 in endothelial progenitor-derived cells under hypoxic conditions. Arthritis Rheum. 58, 3550-3561 (2008).

16. Ballara, S. et al. Raised serum vascular endothelial growth factor levels are associated with destructive change in inflammatory arthritis. Arthritis Rheum. 44, 2055-2064 (2001).

17. Paradowska-Gorycka, A. et al. FLT-1 gene polymorphisms and protein expression profile in rheumatoid arthritis. PLoS ONE 12, e0172018 (2017).

18. Kuryliszyn-Moskal, A., Klimiuk, P. A., Sierakowski, S. \& Ciolkiewicz, M. Vascular endothelial growth factor in systemic lupus erythematosus: relationship to disease activity, systemic organ manifestation, and nailfold capillaroscopic abnormalities. Arch. Immunol. Ther. Exp. (Warsz) 55, 179-185 (2007).

19. Heshmat, N. M. \& El-Kerdany, T. H. Serum levels of vascular endothelial growth factor in children and adolescents with systemic lupus erythematosus. Pediatr. Allergy Immunol. 18, 346-353 (2007).

20. Robak, E., Sysa-Jedrzejewska, A. \& Robak, T. Vascular endothelial growth factor and its soluble receptors VEGFR-1 and VEGFR-2 in the serum of patients with systemic lupus erythematosus. Mediat. Inflamm. 12, 293-298 (2003).

21. Robak, E., Kulczycka, L., Sysa-Jedrzejowska, A., Wierzbowska, A. \& Robak, T. Circulating proangiogenic molecules PIGF, SDF-1 and sVCAM-1 in patients with systemic lupus erythematosus. Eur. Cytokine Netw. 18, 181-187 (2007).

22. Eremina, V. et al. VEGF inhibition and renal thrombotic microangiopathy. N. Engl. J. Med. 358, 1129-1136 (2008).

23. Ostendorf, T. et al. VEGF (165) mediates glomerular endothelial repair. J. Clin. Invest. 104, 913-923 (1999).

24. Eremina, V. et al. Glomerular-specific alterations of VEGF-A expression lead to distinct congenital and acquired renal diseases. J. Clin. Invest. 111, 707-716 (2003).

25. Feliers, D. Vascular endothelial growth factor as a prognostic marker of lupus nephritis. Kidney Int. 75, 1251-1253 (2009).

26. Edelbauer, M. et al. Soluble VEGF receptor 1 promotes endothelial injury in children and adolescents with lupus nephritis. Pediatr. Nephrol. 27, 793-800 (2012).

27. Chirco, K. R. et al. Evaluation of sFLT1 protein levels in human eyes with the FLT1 rs9943922 polymorphism. Ophthalmic Genet. 39, 68-72 (2018).

28. Xiang, W. et al. Evaluating VEGFR1 genetic polymorphisms as a predisposition to AMD in a cohort from northern China. Ophthalmic Genet. 37, 388-393 (2016).

29. Chen, L. et al. Genome-wide assessment of genetic risk for systemic lupus erythematosus and disease severity. Hum. Mol. Genet. https://doi.org/10.1093/hmg/ddaa030 (2020).

30. Fike, A. J., Elcheva, I. \& Rahman, Z. S. M. The post-GWAS era: how to validate the contribution of gene variants in lupus. Curr. Rheumatol. Rep. 21, 3. https://doi.org/10.1007/s11926-019-0801-5 (2019).

31. Hochberg, M. C. Updating the American College of Rheumatology revised criteria for the classification of systemic lupus erythematosus. Arthritis Rheum. 40, 1725-1725 (1997).

32. Arnett, F. C. et al. The American Rheumatism Association 1987 revised criteria for the classification of rheumatoid arthritis. Arthritis Rheum. 31, 315-324 (1988).

33. McCormack, H. M., Horne, D. J. \& Sheather, S. Clinical applications of visual analogue scales: a critical review. Psychol. Med. 18, 1007-1019 (1988).

34. Wallace, S. L. et al. Preliminary criteria for the classification of the acute arthritis of primary gout. Arthritis Rheum. 20, 895-900 (1977).

35. Vitali, C. et al. Classification criteria for Sjogren's syndrome: a revised version of the European criteria proposed by the AmericanEuropean Consensus Group. Ann. Rheum. Dis. 61, 554-558 (2002).

36. van der Linden, S., Valkenburg, H. A. \& Cats, A. Evaluation of diagnostic criteria for ankylosing spondylitis. A proposal for modification of the New York criteria. Arthritis Rheum. 27, 361-368 (1984).

37. Uribe, A. G. et al. The systemic lupus activity measure-revised, the Mexican Systemic Lupus Erythematosus Disease Activity Index (SLEDAI), and a modified SLEDAI-2K are adequate instruments to measure disease activity in systemic lupus erythematosus. J. Rheumatol. 31, 1934-1940 (2004).

\section{Acknowledgements}

This work was supported by grants from the National Natural Science Foundation of China (81701606), Sichuan Provincial Science and Technology Program (2019YJ0540).

\section{Author contributions}

Z.-C.Y. contributed to the data analysis and manuscript editing. J.-M.W. was in charge of sample collection and DNA extraction. Q.W. and J.Z. performed DNA extraction. W.-D.X. and A.-F.H. contributed to ELISA detection and reviewing of the manuscript.

\section{Competing interests}

The authors declare no competing interests.

\section{Additional information}

Supplementary information is available for this paper at https://doi.org/10.1038/s41598-020-72020-8.

Correspondence and requests for materials should be addressed to W.-D.X. or A.-F.H.

Reprints and permissions information is available at www.nature.com/reprints. 
Publisher's note Springer Nature remains neutral with regard to jurisdictional claims in published maps and institutional affiliations.

(c) (i) Open Access This article is licensed under a Creative Commons Attribution 4.0 International License, which permits use, sharing, adaptation, distribution and reproduction in any medium or format, as long as you give appropriate credit to the original author(s) and the source, provide a link to the Creative Commons licence, and indicate if changes were made. The images or other third party material in this article are included in the article's Creative Commons licence, unless indicated otherwise in a credit line to the material. If material is not included in the article's Creative Commons licence and your intended use is not permitted by statutory regulation or exceeds the permitted use, you will need to obtain permission directly from the copyright holder. To view a copy of this licence, visit http://creativecommons.org/licenses/by/4.0/.

(c) The Author(s) 2020 